\title{
Determination of mitochondrial D-loop sequence of Iranian Moghani sheep breed
}

\author{
A Mohammadhashemi ${ }^{1}$, N Pirany ${ }^{1}$, B Baghban Kkohne Roz ${ }^{1}$, M Nassiry $^{2}$ \\ ${ }^{1}$ University of Tabriz, Tabriz, East Azarbaiejan, Islamic Republic of Iran \\ ${ }^{2}$ Ferdowsi University of Mashhad, Mashhad, Khorasan Razavi, Islamic Republic of Iran \\ Email: npirany@gmail.com
}

Introduction The domestic sheep is an important livestock species in many developing countries. They have played a key role in food resources of Iranian plateau which is known as the origin of this species. Sheep are highly adaptable and versatile domestic animals, which has made them a critically important resource in human societies around the word. Molecular genetics have provided valuable information for investigating and conserving genetic resources. Mitochondrial DNA (mtDNA) sequencing has been used to elucidate the complexity and origins of many modern domestic livestock species, leading to a general theme of multiple maternal lineages. The sequence variation existing within mtDNA has proven a particularly useful tool for addressing such questions across a number of livestock species. Till now, four distinct mitochondrial maternal lineages (haplotype groups A, B, C and D) have been found in the domestic sheep.

Material and methods Blood samples from 10 Iranian Moghani sheep were collected from Jafarabad station in Ardabil province. DNA was isolated according to Miller et al. (1988) with minor modifications. The first half of the control region (CR1) region was selected for mtDNA analysis. The PCR products obtained from primers of F:5'TAACTGTGGGGGTAACTATTT-3' and R:5'-AGTATTGAGGACGGGGTAA-3' were purified and then sequenced (MWG-BIOTECH AG, Germany). The sequences obtained were aligned and edited using Bioedit (Hall 1999). All similar sequences available were obtained from Genebank (NCBI) and phylogenic relationships were drawn using MEGA4 (Tamura et al 2007).

Results After analyzing the sequences obtained, the length of the CR1 region in Moghani sheep was found to be around 420 base pairs. We obtained SNPs and 5 haplotypes (Table 1). All haplotypes were of the (Asian) A type. NeighbourJoining tree of 29 mitochondrial sheep including the Mouflon, a wild sheep and European sheep was constructed. The tree contained four distinct branches: clades A, B, C and D. The Iranian Moghani sheep carrying A haplotype, was clustered in the A clade with another Iranian sheep breed, the Baluchi and one Turkish breed which was expected because of their geographical closeness. The branch style tree was drawn for analyzing samples in A haplotype group and results suggested that Ovis urial is the origin of this breed. Finally, the consensus sequence was registered in NCBI under accession number FJ531545.

Table 1 Haplotype sequences in Moghani sheep breed

\begin{tabular}{lllllllllll}
\hline \hline Sample & Haplotype & $56^{*}$ & 75 & 83 & 90 & 303 & 314 & 318 & 322 & 327 \\
\hline Sample 1 & 1 & $\mathrm{C}$ & $\mathrm{T}$ & $\mathrm{T}$ & $\mathrm{T}$ & $\mathrm{T}$ & $\mathrm{C}$ & $\mathrm{T}$ & $\mathrm{A}$ & $\mathrm{A}$ \\
Sample 2 & 2 & $\mathrm{~A}$ & $\mathrm{~T}$ & $\mathrm{C}$ & $\mathrm{C}$ & $\mathrm{C}$ & $\mathrm{T}$ & $\mathrm{C}$ & $\mathrm{G}$ & $\mathrm{G}$ \\
Sample 3 & 3 & $\mathrm{~A}$ & $\mathrm{C}$ & $\mathrm{T}$ & $\mathrm{T}$ & $\mathrm{T}$ & $\mathrm{C}$ & $\mathrm{C}$ & $\mathrm{A}$ & $\mathrm{A}$ \\
Sample 4 & 4 & $\mathrm{~A}$ & $\mathrm{~T}$ & $\mathrm{~T}$ & $\mathrm{~T}$ & $\mathrm{~T}$ & $\mathrm{C}$ & $\mathrm{T}$ & $\mathrm{A}$ & $\mathrm{A}$ \\
Sample 5 & & $\mathrm{A}$ & $\mathrm{T}$ & $\mathrm{T}$ & $\mathrm{T}$ & $\mathrm{T}$ & $\mathrm{C}$ & $\mathrm{T}$ & $\mathrm{A}$ & $\mathrm{A}$ \\
Sample 6 & 5 & $\mathrm{~A}$ & $\mathrm{~T}$ & $\mathrm{~T}$ & $\mathrm{~T}$ & $\mathrm{C}$ & $\mathrm{C}$ & $\mathrm{C}$ & $\mathrm{A}$ & $\mathrm{A}$ \\
Sample 7 & & $\mathrm{A}$ & $\mathrm{T}$ & $\mathrm{T}$ & $\mathrm{T}$ & $\mathrm{C}$ & $\mathrm{C}$ & $\mathrm{C}$ & $\mathrm{A}$ & $\mathrm{A}$ \\
Sample 8 & & $\mathrm{~A}$ & $\mathrm{~T}$ & $\mathrm{~T}$ & $\mathrm{~T}$ & $\mathrm{C}$ & $\mathrm{C}$ & $\mathrm{C}$ & $\mathrm{A}$ & $\mathrm{A}$ \\
Sample 9 & & $\mathrm{A}$ & $\mathrm{T}$ & $\mathrm{T}$ & $\mathrm{T}$ & $\mathrm{C}$ & $\mathrm{C}$ & $\mathrm{C}$ & $\mathrm{A}$ & $\mathrm{A}$ \\
Sample 10 & & $\mathrm{~A}$ & $\mathrm{~T}$ & $\mathrm{~T}$ & $\mathrm{~T}$ & $\mathrm{C}$ & $\mathrm{C}$ & $\mathrm{C}$ & $\mathrm{A}$ & $\mathrm{A}$ \\
\hline \hline
\end{tabular}

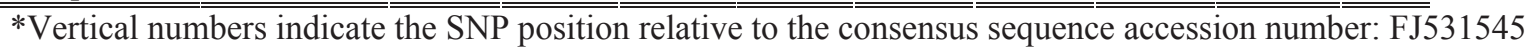

Conclusions This finding may arise from high levels of relatedness and a low effective population size (Ne) within the animals sequenced. Sequence analysis of the mtDNA from Moghani breed with another Iranian sheep showed high genetic diversity within some Iranian sheep breeds. However, more data from sheep breeds need to be analyzed to confirm these findings

\section{References}

Miller, S.A., Dykes, D.D. and Polesky, H.F. 1998. Nucleic Acid Research 16, 12-15.

Hall, T.A. 1999. Nucleic Acids Symposium Series 4, 95-98.

Tamura, K., Dudley, J., Nei, M. and Kumar, S. 2007. Molecular Biology and Evolution 24, 1596-1599. 